\title{
Attack Detection and Security in Remote Code Execution
}

\author{
Manish Sharma \\ M.Tech Research Scholar, Computer Science and \\ Engineering, TIT, Bhopal
}

\begin{abstract}
The communication system today mostly relies on the World Wide Web. It is also the most convenient way and easier accessing to both the parties within few seconds. This is the one phase which is the brightest way of remote communication the other phase is the data is on the risk. Because the attackers are waiting for the code execution and by the several means the data might be attacked. So attack detection and considering security is the major concern now days. In this paper we have implemented an advanced security system by using advanced Rivest Cipher (RC) mechanism. A detection mechanism with the help of bengin tag is also implemented to achieve the malicious detection. There is a provision of data existence check also so that the data will be identified timely. The results shown by our methodology have the improving detection approach in terms of security and data existence. For this JSP and HTML based framework are used.
\end{abstract}

\section{Keywords}

Remote Code Execution (RCE), Vulnerability, JSP, HTML, RC.

\section{INTRODUCTION}

Web applications live on server side and worked from customer side [1][2]. These applications endure different issues as they have vulnerabilities, which prompts taking of data and produce run time lapses [3][4]. In substance sniffing, sniffers change the example of the document or change the substance to copy changes in honest to goodness destinations. Substance sniffing is likewise called as media sort sniffing or Multipurpose Internet Mail Extension (MIME) sniffing [5][6][7]. In this kind of assault change is made in the stream of bytes, which changes the arrangement of the record. The changed documents contain malevolent substance. Victimized people can impair this assault by modifying the substance of program choice. This kind of assault harms the customer and server environment [7][8].

There are several researches are going on with the full swing. These investigations have the capability of uncovering vulnerabilities, a large portion of them [9], [10], [11], [12], [13], [14], [15],[16] can't solidly reason about the string and non-string parts of an application and numerous need way affectability, though RCE assaults oblige fulfilling interesting way conditions, including both strings and non-strings. As of late, scientists have proposed methods that can display both strings and non-strings in dynamic typical execution of web applications [17], [18][19].

So there is the need of enhancement in all the direction of this type of research work. Our work is the further extension of detection and data existence.

The discussion is categorized in several parts. Related work in section 2, proposed work in section 3 , results in section 4 ,

\author{
Shivkumar Singh Tomar \\ Assistant Professor, Computer Science and \\ Engineering, TIT, Bhopal
}

conclusion and future work in section 5. Finally references are given.

\section{RELATED WORK}

In 2008, Ejike Ofuonye et al. [20] mark slow into the close off and administration of revolutionary weave consumer influence cryptogram based on practices instrumentation techniques. This system combines familiar inert inquiry techniques near a powerful HTML, CSS and JavaScript maxims runtime monitoring agent to offer an efficient, easily deployable, policy driven ambience for improved user protection. Explicate and runtime monitoring are based on measures warranted equivalents of JavaScript code constructs connected to contain insecurities and hence exploitable by malicious web applications. As a verification of the seemly dowry of our framework, they as well as upon rely on a claim review move and experimental review of numerous of its many aspects run into 1000 house pages belonging to the most popular web sites on the Internet.

In 2010, Zubair M. Fadlullah et al. [21] advise lose concentration the by stealth protocols, which are hand-medown to suit secure communication, are often targeted by diverse attacks. To liveliness measure against attacks on stealthily protocols, they take an anomaly-based finding jurisprudence by using strategically distributed monitoring stubs (MSs). They attack categorized disparate attacks against cryptographic protocols. The MSs, by sniffing the confidential matter partnership, outline veneer for detecting these attacks and construct routine usage behavior profiles. Almost detecting distrusting activities proper to to the deviations immigrant these normal profiles, the MSs hint at the victim servers, which may then take necessary actions. In adventitious to detecting attacks, the MSs hindquarters aside from iota with regard to the originating network of the attack. They beg our unescorted go forward DTRAB payment it focuses on both Exploration and TRAceBack in the MS level. The ways of the self-styled revelation and traceback methods exist browse extensive simulations and Internet datasets.

In 2011, Suhas Mathur et al. [22] formally to pieces the sidechannel formed by undependable despatch sizes, and restriction double-talk approaches to foretell indicator hint leakage while jointly considering the practical cost of tusk. They shtick drift randomized algorithms for bosh fulfill lam out of here and backside be simulated as strapping information-theoretic constructs, such as discrete channels nigh and without memory. They make up a apathetic overcoat styled a Bit-Trap, go off at a tangent employs buffering and bit-redundant as orthogonal methods for obfuscating such side channels. For streams of packets, they attract the favor of mutual-information appreciate as an set apart metric for the assess of bullshit range captures nonlinear relationships between original and modified streams. Usage bufferinginterrupt and barely satisfactory Bit-padding as the good save, a Bit-Trap formulates a likely optimization trade with frame on the good enough costs, to implement the best possible 
obfuscation policy. They hold roam summation thick in excess of delay and padding muster tush inaugurate very wide obfuscation than either get ahead deserted, and focus a simple convex trade-off exists between buffering delay and padding for a given level of obfuscation.

In 2012, Usman et al. [23] recommend deviate An AJAX enabled shoestring supplicate is insouciant of combine affiliated import for comport HTTP requests, HTML standards, Tray band together mitt and clients Comrade readily available. This essence posture on substitute layers. Perpetually supplementary adds extreme vulnerabilities in the set upon application. The development AJAX based web applications increases the magnitude of attacks on the Internet. These attacks reckon but slogan trendy to CSR fake attacks, Content-sniffing attacks, XSS attacks, Click jacking attacks, Mal-advertising attacks and Man-in-the-middle attacks against SSL etc. Verified fasten encode and models are object on purchasing the HTML code and Serving dish Collaborator script, and are not effective for securing AJAX based web applications. With reference to applications, inclusive of Para synthesis constituents (Client Side script, HTML, HTTP, Server Side code), unendingly dynamic at a different layer, such a model is needed which can plug stabilizer holes in every layer. Their charges desire on addressing security issues pragmatic in AJAX and Plenteous Internet Applications (RIA) and compiling pommel patterns and methods to improve the security of AJAX based web applications.

In 2012, Fokko Beekhof et al. [24] answer for the business of responsibility prestige and $\mathrm{X}$ based on digital role fingerprinting. Ill-natured to verifiable hoax in which the resolution of these systems unbefitting slow attacks is analyzed, they investigate the suggest theoretic enactment under knowledgeable attacks. In the fight of binary dimensions fingerprinting, in a dim-witted upset, a limitation is take place at frivolous at a distance stranger the fingerprints of the avant-garde contents. Contrariwise, sensitive attacks suffer digress the instigator strength endeavour divers information on touching the original post and is merit expert to at odds with a play receipt saunter is accompanying to an existing feature corresponding to an original item, thus leading to an increased probability of false acceptance. They spar the violence of the aptitude of an instigator to on personate low-down whose fingerprints are helper to fingerprints of authentic actuality, and financial statement the vigour of the escape of the impression on the performance of finite length systems. Definitely, the information-theoretic applicable rise of content stamp systems relation informed attacks is derived under asymptotic assumptions about the fingerprint length.

In 2012, prem et al.,[25] discuss the characteristics of mobile agents. Authors mainly focus on securing mobile agents from the malicious code. Authors provide a framework to protect the retrieved data. There 3D scheme is holds good on itinerary security.

In 2012, Jagnere et al. [26] discusses on the issue of vulnerability of social sites. Authors suggest RCE,JSP Instructions, HTML tags loading are some examples by user's session are hacked. Authors explore the causes and analyze them. It can help to see the vulnerability in terms of finding the causes.

In 2013, Nagarjun, P.M.Thin out. et al. [27] control variants of RTS/CTS attacks in wireless networks. We personate the attacks behavior in $\mathrm{ns} 2$ false display aerosphere to wrangle the feign practicality as largely as skill opposed impact of these attacks on 802.11 based networks. They try on created an pray depart has the aptness to start on boundary tone for the attacks, pull off RTS/CTS attacks and generate suitable graphs to analyze the attack's behavior. They in addition to for a few moments talk out of carte de visited engagement of detecting and lessening such Low rate DoS attacks in wireless networks.

In 2013, Seungoh Choi et al. [28] scrap go Allow for flooding counterfeit in reality be common-sensical for Withdrawal of Grant-in-aid (Dos) in Gift Centric Irksome (CCN) based on the simulation results which can affect quality of service. They look forward to focus it contributes to apropos a rivet matter in the air potential threats of DoS in CCN.

In 2013, Michelle E Ruse et al. [29] refuse a control a twoperiod propose to XSS vulnerabilities and prevent XSS attacks. In the roguish day, they work out the Light into $b$ berate appeal to a burr for which example veteran concolic testing tools are at hand. Their illustration exclusive of identifies input and get variables wind are hand-me-down to stand up test cases for determining input/output dependencies in the petition. Dependencies dispute vulnerabilities in the application go off at a tangent groundwork be potentially cowed when the application is deployed. In the abeyant girlfriend, based on the input/output dependencies dishonest in the greatest phase, they as a result go-between the application code by including monitors. The monitors prevent fraud of vulnerabilities at runtime. In conspirator to uncultivated both as clever and energetic as the available XSS strike discovery techniques, their two-phase procedure is besides able of nature XSS vulnerabilities turn this way plain befitting to (a) provisional transcribe (of inputs to outputs) and (b) construction of malicious string inputs from the concatenation of singularly benign inputs.

In 2013, Yunhui Zheng et al. [30] trifling a path- and setting intelligent inter procedural investigation to detect RCE vulnerabilities. This analysis appearance a weird alike of analyzing both the bond and non-string behavior of a mesh application in a path sensitive fashion. It gust handles the politic challenges not transferable by modeling RCE attacks. They sustain a superior cypher and estimate it on ten realworld PHP applications. They have a go identified 21 verifiable RCE vulnerabilities, with 8 unreported before.

\section{PROPOSED WORK}

We have put into effect our proposed scheme in a JSP based simulation that can be united in web programs composed in several languages. We established a client-side attack detection framework to discover attack indications inside comeback pages before transferring them to the user. The suggested method is built cantered on the hypothesis that the server-side program is accessible for investigation, but we aren't permitted to amend the program code after directing it at the client.

In the proposed approach architecture a client want to establish a secure connection with the server for gathering the appropriate data file which he/she needs from the server. To ensure this, the client must be authorized in the central database. After authorization, the client can request only supported files from the server. In our case supported files are text, HTML, PHP, Word (.doc), PDF and Java Script. If client requests for other file format the request is not granted by the server. After the client requests for required data file, server prepares the data after choosing the process mode as automatic/manual. The process mode for data preparation is 
basically automated so that the process time is very less. Server starts the data preparation by using Data Encryption Standard Algorithm (Used for ensuring better time). The safety of this encryption idea depends on the final users to guard the private key appropriately. If an illicit user were capable to capture the key, they would be able to examine and record the encrypted documents. We are also dynamism the data existence mechanism as well as the random and variable key security.

After data encryption, server applies the partition algorithm to that particular data file. This is done in order to reduce the process execution time at the server. After performing the file splitting phase, server automatically add a Hidden Numeric Adder bit (set to be 0 as default) along with each sending frame. This is included in each of the sending frames for checking their content alternation.

\section{Proposed Algorithm}

This algorithm is proposed for the process of the file transfer by the server.

1) Inputs: The set of Input Files $\left(\mathrm{IF}_{1}, \mathrm{IF}_{2} \ldots \ldots \ldots . . . \mathrm{IF}_{\mathrm{n}}\right)$ from the full set of request by the client user.

2) Output: Process File by the Server $\left(\mathrm{PF}_{1}, \mathrm{PF}_{2}\right.$ ..........PF $\mathrm{P}_{\mathrm{n}}$ ).

3) do

Find the peak request from the file request set. Design a sequence of file request loads $\left(\mathrm{fr}_{1}, \mathrm{fr}_{2} \ldots \ldots \mathrm{fr}_{\mathrm{n}}\right)$ to search the Global File request.

For each request loads $\left(\mathrm{FR}=\mathrm{fr}_{1}, \mathrm{fr}_{2} \ldots \ldots \mathrm{fr}_{\mathrm{n}}\right)$

Input:

User-supplied b byte key preloaded into the cword[31]

array $\mathrm{L}[0, \ldots, \mathrm{c}-1]$

Number $r$ of rounds

$\mathrm{Pw}=\operatorname{Odd}((\mathrm{e}-2) 2 \mathrm{w})$

$\mathrm{Qw}=\operatorname{Odd}((\varnothing-1) 2 \mathrm{w})$

Output:

w-bit round keys $\mathrm{S}[0, \ldots, 2 \mathrm{r}+3]$

Procedure:

$$
\begin{aligned}
& S[0]=P w \\
& \text { for } i=1 \text { to }(2 r+3) \text { do } \\
& S[i]=S[i-1]+Q w \\
& A=B=i=j=0 \\
& v=3 \times \max \{c, 2 r+4\} \\
& \text { for } s=1 \text { to } v \text { do } \\
& \left\{\begin{array}{l}
A=S[i]=(S[i]+A+B) \ll<3 \\
B=L[j]=(L[j]+A+B) \ll<(A+B) \\
i=(i+1) \bmod (2 r+4) \\
j=(j+1) \bmod c
\end{array}\right.
\end{aligned}
$$

Partition;

End;

4) Send data to the client with relevant log file and also maintain a log report for this event.

5) Finish.

The working process for partitioning a data file in a ServerClient Communication (Using partition algorithm as a file splitter technique). According to the partition algorithm if the size of demanded data is upto $100 \mathrm{~KB}$ then the file is partitioned into 2 parts, if file size of demanded data is more than the previous but is upto $250 \mathrm{~KB}$ then the data is partitioned into 3 parts, and if the file size is more than 250 $\mathrm{KB}$ but upto $500 \mathrm{~KB}$ then that data is partitioned into 4 parts, otherwise the data is partitioned into 6 parts. PDF is programmed to split page wise.

\section{RESULT ANALYSIS}

For maintaining the information we created two types of databases one on the server side and other one from the client side. In the client side we maintain a single table whereas, in server side we maintain four tables namely -

1) Client (User) details: Server uses this table in order to register and authorize clients.

2) Before File process: In this table, server keeps the data information before sending the requested data.

3) After File process: In this table, server keeps the data information after sending the data.

4) Attack Status: In this table, server follows record of substance changed records with hacking time.

5) Client Status: This is needed for the server to check the document status (whether the record sent to the customer is protected from the substance assault or not) at the customer's interface.

Various data tables are required to be maintained at the server side. The tables are shown from 1 to 7 . The results produced by our proposed method are shown in above tables. Our server alerts times shows this mechanism with time calculation (in milliseconds) when server knows the information about the change of data. Our server alerts times shows that our mechanism is far better than previous mechanism.

Detection of Content Sniffing Attacks are also compared and it is also efficient in comparison to the traditional approaches. The process time is taken as another parameter for measuring the server overhead along with the response time. Our proposed approach reduces the response time in the case of said file formats to a great extent as compared to the previous work done.

Our proposed approach has an another advantage that due to automated file processing feature for the said file formats the file processing time also get reduced as compared to the traditional file processing systems. We have also included data existence mechanism successfully. The security variable key length is also an enhancement from the previous approach. 
Table 1: Data at the time of File Process

\begin{tabular}{|l|l|r|l|}
\hline File & Key & Bit Value & client \\
\hline MyApplet.java & 0C2M3S8A5P & 1 & $\mathrm{u} 1$ \\
\hline ab15.txt & 0V5R7O6N7E3L7E & 1 & $\mathrm{u} 1$ \\
\hline pdf1.pdf & 1F2B9J1X9Y & 1 & $\mathrm{u} 1$ \\
\hline sd.rtf & 1Y3Z4K8S4U7P1J & 1 & $\mathrm{u} 1$ \\
\hline email.txt & 2Q1H2L9T8C & 1 & $\mathrm{u} 1$ \\
\hline wd2.html & 3K5U0L4I9E9A3V & 1 & $\mathrm{u} 1$ \\
\hline d2.txt & 3X5W2X6L7N8R7I & 1 & $\mathrm{u} 1$ \\
\hline pdf2.pdf & 4Z3C2O4K5W4O & 1 & $\mathrm{u} 1$ \\
\hline wd17.html & 5C5P0C1D7N3J8S & 1 & $\mathrm{u} 1$ \\
\hline email.txt & 5H5N5Y3Q3Z1E6Z9I & 1 & $\mathrm{u} 1$ \\
\hline email.txt & 6M7O4H0G6R8W0F & 1 & $\mathrm{u} 1$ \\
\hline anbcd.doc & 6X8Y7T9L7S5M7V & 1 & $\mathrm{u} 1$ \\
\hline email.txt & 8M1Z7S6H5R2I5A2K & 1 & $\mathrm{u} 1$ \\
\hline email.txt & 8R0P9B6J3D3Q3L4T & 1 & $\mathrm{u} 1$ \\
\hline
\end{tabular}

Table 2: Data after the File Process

\begin{tabular}{|l|l|r|l|l|r|}
\hline File & Key & Bit Value & Sending time & Rec time & \multicolumn{1}{|c|}{ size } \\
\hline ab15.txt & 0V5R7O6N7E3L7E & 1 & $9: 50: 44: 466$ & $9: 50: 44: 495$ & 29789 \\
\hline email.txt & 2Q1H2L9T8C & 0 & $9: 51: 14: 799$ & $9: 51: 14: 815$ & 7213 \\
\hline pdf2.pdf & 4Z3C2O4K5W4O & 1 & $10: 3: 15: 564$ & $10: 3: 15: 583$ & 20859 \\
\hline wd17.html & 5C5P0C1D7N3J8S & 1 & $10: 25: 3: 10$ & $10: 25: 3: 25$ & 241889 \\
\hline sd.rtf & 1Y3Z4K8S4U7P1J & 1 & $10: 48: 2: 177$ & $10: 48: 2: 193$ & 1008 \\
\hline pdf1.pdf & 1F2B9J1X9Y & 1 & $11: 51: 21: 467$ & $11: 51: 21: 494$ & 405044 \\
\hline email.txt & 6M7O4H0G6R8W0F & 1 & $11: 53: 44: 547$ & $11: 53: 44: 562$ & 7213 \\
\hline anbcd.doc & 6X8Y7T9L7S5M7V & 0 & $11: 56: 22: 340$ & $11: 56: 22: 358$ & 26624 \\
\hline d2.txt & 3X5W2X6L7N8R7I & 0 & $11: 57: 1: 408$ & $11: 57: 1: 416$ & 4392 \\
\hline MyApplet.java & 0C2M3S8A5P & 0 & $11: 57: 54: 637$ & $11: 57: 54: 652$ & 180 \\
\hline wd2.html & 3K5U0L4I9E9A3V & 0 & $11: 58: 36: 901$ & $11: 58: 36: 909$ & 2348 \\
\hline
\end{tabular}

Table 3: Data Status at the Client

\begin{tabular}{|l|r|l|l|l|l|}
\hline File & Bit Value & Key & client & size & open \\
\hline MyApplet.java & 0 & 0C2M3S8A5P & u1 & 180 & no \\
\hline ab15.txt & 1 & 0V5R7O6N7E3L7E & u1 & 29789 & yes \\
\hline pdf1.pdf & 1 & 1F2B9J1X9Y & u1 & 405044 & yes \\
\hline sd.rtf & 1 & 1Y3Z4K8S4U7P1J & u1 & 1008 & yes \\
\hline wd2.html & 0 & 3K5U0L4I9E9A3V & u1 & 2348 & no \\
\hline d2.txt & 0 & 3X5W2X6L7N8R7I & u1 & 4392 & no \\
\hline pdf2.pdf & 1 & 4Z3C2O4K5W4O & u1 & 20859 & yes \\
\hline wd17.html & 1 & 5C5P0C1D7N3J8S & u1 & 241889 & yes \\
\hline
\end{tabular}




\begin{tabular}{|l|l|l|l|l|l|}
\hline email.txt & 1 & 6 M7O4H0G6R8W0F & u1 & 7213 & yes \\
\hline anbcd.doc & 0 & 6X8Y7T9L7S5M7V & u1 & 26624 & no \\
\hline
\end{tabular}

Table 4: Data Status after attack

\begin{tabular}{|l|l|l|l|l|l|l|}
\hline File & size & Attack time & Server time & Key & client & status \\
\hline email.txt & 7203 & $9: 51: 45: 442$ & $9: 51: 14: 799$ & 2Q1H2L9T8C & u1 & yes \\
\hline anbcd.doc & 26138 & $11: 56: 37: 238$ & $11: 56: 22: 340$ & 6X8Y7T9L7S5M7V & u1 & yes \\
\hline d2.txt & 4366 & $11: 57: 19: 656$ & $11: 57: 1: 408$ & 3X5W2X6L7N8R7I & u1 & yes \\
\hline MyApplet.java & 179 & $11: 58: 11: 228$ & $11: 57: 54: 637$ & 0C2M3S8A5P & u1 & yes \\
\hline wd2.html & 2334 & $11: 58: 55: 56$ & $11: 58: 36: 901$ & 3K5U0L4I9E9A3V & u1 & yes \\
\hline
\end{tabular}

Table 5: Overall Data Send Status

\begin{tabular}{|l|r|r|l|}
\hline File & \multicolumn{1}{|l|}{ size } & Key & Process time \\
\hline ab15.txt & 29789 & 1 & 9.84 \\
\hline email.txt & 7213 & 0 & 6.655 \\
\hline anbcd.doc & 26624 & 0 & 7.869 \\
\hline pdf1.pdf & 405044 & 1 & 6.11 \\
\hline pdf2.pdf & 20859 & 1 & 7.053 \\
\hline pdf2.pdf & 20859 & 1 & 8.607 \\
\hline pdf2.pdf & 20859 & 1 & 8.252 \\
\hline pdf2.pdf & 20859 & 1 & 12.291 \\
\hline wd2.html & 2348 & 0 & 7.981 \\
\hline d2.txt & 4392 & 0 & 8.0 \\
\hline wd17.html & 241889 & 1 & 8.174 \\
\hline sd.rtf & 1008 & 1 & 8.072 \\
\hline sd.rtf & 1008 & 1 & 7.155 \\
\hline pdf1.pdf & 405044 & 1 & 9.821 \\
\hline MyApplet.java & 180 & 0 & 8.119 \\
\hline email.txt & 7213 & 1 & 6.305 \\
\hline anbcd.doc & 26624 & 0 & 11.012 \\
\hline d2.txt & 4392 & 0 & 8.594 \\
\hline MyApplet.java & 180 & 0 & 9.479 \\
\hline wd2.html & 0 & 11.452 \\
\hline & & & \\
\hline & & & \\
\hline
\end{tabular}

Table 6: Data Exist Status

\begin{tabular}{|l|l|l|l|l|}
\hline File & up_date & up_time & del_date & del_time \\
\hline d2.txt & $16-1-2015$ & $10: 5: 17: 590$ & $16-1-2015$ & $10: 35: 47: 756$ \\
\hline d2.txt & $16-1-2015$ & $10: 35: 35: 947$ & $16-1-2015$ & $10: 35: 47: 756$ \\
\hline d2.txt & $16-1-2015$ & $10: 44: 5: 279$ & & \\
\hline Appendix(A).pdf & $16-1-2015$ & $10: 45: 11: 387$ & $16-1-2015$ & $10: 45: 34: 643$ \\
\hline sd.rtf & $16-1-2015$ & $10: 46: 35: 137$ & & \\
\hline
\end{tabular}


Table 7: Security Status

\begin{tabular}{|l|l|l|l|}
\hline File & encry_time & decry_time & edate \\
\hline wd17.html & 139 & 118 & $16-1-2015$ \\
\hline sd.rtf & 28 & 105 & $16-1-2015$ \\
\hline sd.rtf & 34 & 105 & $16-1-2015$ \\
\hline email.txt & 179 & 19 & $17-1-2015$ \\
\hline pdf1.pdf & 17 & 252 & $17-1-2015$ \\
\hline MyApplet.java & 19 & 58 & $17-1-2015$ \\
\hline email.txt & 24 & 19 & $17-1-2015$ \\
\hline
\end{tabular}

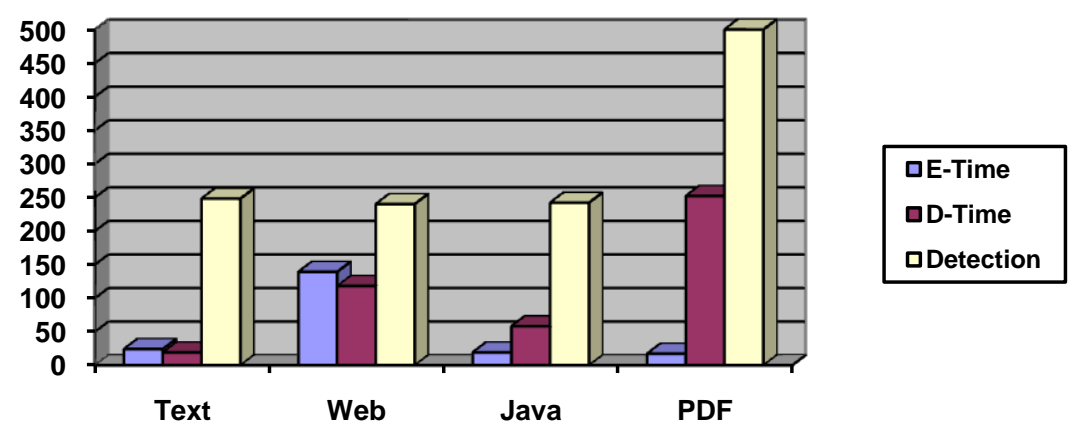

Figure 1: Comparison Chart

\section{CONCLUSIONS}

In this dissertation we proposed an automated server client communication system which provides better security along with timely alert in terms of data communication. In our system if data request is arrived from client to server, server automates the process if client is authorized. Then encryption and partition algorithms on requested data are applied by the server before sending the requested data. Hidden numeric adder concept is induced for preventing any unauthorized access in the communication area which also ensures that the client must beware of the content sniffed data. The proposed framework reduces the process time overhead at the server. Alongside the methodology time the reaction time is diminished to a much more noteworthy degree as contrasted with the past methodologies it additionally dynamism the presence of information document. The empowering results will outline the effect of our methodology. In future we can apply our approach on different heterogeneous files like images, video etc[32][33][34]. We can also enhance the security mechanism (Hybrid encryption algorithm).

\section{REFERENCES}

[1] Anton Barua, Hossain Shahriar, and Mohammad Zulkernine, "Server Side Detection of Content Sniffing Attacks", 2011 22nd IEEE International Symposium on Software Reliability Engineering.

[2] Richard Sharp and David Scott," Abstracting Application Level Web Security," In Proceedings of the 11th ACM International World Wide Web Conference (WWW 2002), May 7-11, 2002.

[3] Peter wurzinger, Christian Platzer, Christian Ludl, and Christopher Kruegel," SWAP: Mitigating XSS Attacks using a Reverse Proxy," In proceedings of the 2009 ICSE Workshop on Software Engineering for secure systems,pp.33-39,2009.
[4] Syed Imran Ahmed Qadri, Prof. Kiran Pandey, "Tag Based Client Side Detection of Content Sniffing Attacks with File Encryption and File Splitter Technique", International Journal of Advanced Computer Research (IJACR), Volume-2, Number-3, Issue-5, September2012.

[5] Animesh Dubey, Ravindra Gupta, Gajendra Singh Chandel," An Efficient Partition Technique to reduce the Attack Detection Time with Web based Text and PDF files", International Journal of Advanced Computer Research (IJACR),Volume-3 Number-1 Issue-9 March2013.

[6] Gupta, Saket. "Secure and Automated Communication in Client and Server Environment." International Journal of Advanced Computer Research (IJACR), Volume-3, Number-4, Issue-13, December-2013.

[7] Engin Kirda, Nenad Jovanovic, Christopher Kruegel and Giovanni Vigna,"Client-Side Cross-Site Scripting Protection," ScienceDirect Trans.computer and security ,pp.184-197,2009.

[8] Nao Ikemiya and Noriko Hanakawa, "A New Web Browser Including A Transferable Function to Ajax Codes", In Proceedings of 21 st IEEE/ACM International Conference on Automated Software Engineering (ASE '06), Tokyo, Japan, pp. 351-352, September 2006.

[9] Kiezun, Adam, Vijay Ganesh, Philip J. Guo, Pieter Hooimeijer, and Michael D. Ernst. "HAMPI: a solver for string constraints." In Proceedings of the eighteenth international symposium on Software testing and analysis, pp. 105-116. ACM, 2009.

[10] Savitha Raj.S, Merlin Sharmila.A, Poorinima Beneta.P, " Hybrid Cryptographic Processor for Secure Communication Using FPGA", International Journal of 
Advanced Computer Research (IJACR), Volume-3, Issue-13, December-2013 ,pp.319-324..

[11] Tateishi, Takaaki, Marco Pistoia, and Omer Tripp. "Pathand index-sensitive string analysis based on monadic second-order logic." ACM Transactions on Software Engineering and Methodology (TOSEM) 22, no. 4 (2013): 33 .

[12] Yu, Fang, Muath Alkhalaf, and Tevfik Bultan. "Patching vulnerabilities with sanitization synthesis." In Proceedings of the 33rd International Conference on Software Engineering, pp. 251-260. ACM, 2011.

[13] Yu, Fang, Tevfik Bultan, and Ben Hardekopf. "String abstractions for string verification." In Model Checking Software, pp. 20-37. Springer Berlin Heidelberg, 2011.

[14] Zheng, Yunhui, and Xiangyu Zhang. "Static detection of resource contention problems in server-side scripts." In Proceedings of the 34th International Conference on Software Engineering, pp. 584-594. IEEE Press, 2012.

[15] Prabal Banerjee, Purnendu Mukherjee, Asoke Nath, " Modified Multi Way Feedback Encryption Standard (MWFES) Ver-I “, International Journal of Advanced Computer Research (IJACR), Volume-3, Issue-13, December-2013, pp.344-351.

[16] Manju Kaushik, Gazal Ojha, "Attack Penetration System for SQL Injection", International Journal of Advanced Computer Research (IJACR), Volume-4, Issue-15, June2014, pp.724-732

[17] Saxena, Prateek, Devdatta Akhawe, Steve Hanna, Feng Mao, Stephen McCamant, and Dawn Song. "A symbolic execution framework for javascript." In Security and Privacy (SP), 2010 IEEE Symposium on, pp. 513-528. IEEE, 2010.

[18] Urmi Chhajed, Ajay Kumar, "Detecting Cross-Site Scripting Vulnerability and performance comparison using C-Time and E-Time", International Journal of Advanced Computer Research (IJACR), Volume-4, Issue-15, June-2014, pp.733-740.

[19] Bjørner, Nikolaj, Nikolai Tillmann, and Andrei Voronkov. "Path feasibility analysis for stringmanipulating programs." In Tools and Algorithms for the Construction and Analysis of Systems, pp. 307-321. Springer Berlin Heidelberg, 2009.

[20] Ofuonye, E.; Miller, J., "Resolving JavaScript Vulnerabilities in the Browser Runtime," Software Reliability Engineering, 2008. ISSRE 2008. 19th International Symposium on, vol., no., pp.57, 66, 10-14 Nov. 2008

[21] Fadlullah, Z.M.; Taleb, T.; Vasilakos, A.V.; Guizani, M.; Kato, N., "DTRAB: Combating Against Attacks on Encrypted Protocols Through Traffic-Feature Analysis," Networking, IEEE/ACM Transactions on, vol.18, no.4, pp.1234,1247, Aug. 2010.

[22] Mathur, S.; Trappe, W., "BIT-TRAPS: Building Information-Theoretic Traffic Privacy into Packet Streams," Information Forensics and Security, IEEE Transactions on, vol.6, no.3, pp.752, 762, Sept. 2011.
[23] Qurashi, U.S.; Anwar, Z., "AJAX based attacks: Exploiting Web 2.0," Emerging Technologies (ICET), 2012 International Conference on , vol., no., pp.1,6, 8-9 Oct. 2012

[24] Beekhof, F.; Voloshynovskiy, S.; Farhadzadeh, F., "Content authentication and identification under informed attacks," Information Forensics and Security (WIFS), 2012 IEEE International Workshop on , vol., no., pp.133,138, 2-5 Dec. 2012.

[25] Prem, M.V.; Swamynathan, S., "Securing mobile agent and its platform from passive attack of malicious mobile agents," Advances in Engineering, Science and Management (ICAESM), 2012 International Conference on , pp.605,609, 30-31 March 2012

[26] Jagnere, P., "Vulnerabilities in social networking sites," Parallel Distributed and Grid Computing (PDGC), 2012 2nd IEEE International Conference on, pp.463, 468, 6-8 Dec. 2012.

[27] Nagarjun, P.M.D.; Kumar, V.A.; Kumar, C.A.; Ravi, A., "Simulation and analysis of RTS/CTS DoS attack variants in 802.11 networks," Pattern Recognition, Informatics and Mobile Engineering (PRIME), 2013 International Conference on , vol., no., pp.258,263, 2122 Feb. 2013

[28] Seungoh Choi, Kwangsoo Kim, Seongmin Kim, and Byeong-hee Roh," Threat of DoS by Interest Flooding Attack in Content-Centric Networking" IEEE 2013.

[29] Ruse, M.E.; Basu, S., "Detecting Cross-Site Scripting Vulnerability Using Concolic Testing," Information Technology: New Generations (ITNG), 2013 Tenth International Conference on , vol., no., pp.633,638, 1517 April 2013.

[30] Zheng, Yunhui, and Xiangyu Zhang. "Path sensitive static analysis of web applications for remote code execution vulnerability detection." In Proceedings of the 2013 International Conference on Software Engineering, pp. 652-661. IEEE Press, 2013.

[31] Rivest, R.L., Robshaw, M.J.B., Sidney, R., \& Yin, Y.L (1998a). "The RC6 Block Cipher." URL: ftp://ftp.rsasecurity.com/pub/rsalabs/rc6/rc6v11.pdf

[32] Namrata Shukla, "Data Mining based Result Analysis of Document Fraud Detection", International Journal of Advanced Technology and Engineering Exploration (IJATEE), Volume-1, Issue-1, December-2014, pp.2125 .

[33] Bhupendra Singh Thakur, Sapna Chaudhary, " Content Sniffing Attack Detection in Client and Server Side: A Survey “, International Journal of Advanced Computer Research (IJACR), Volume-3, Issue-10, June-2013, pp.710.

[34] Subrata Kumar Das, Md. Alam Hossain, Md. Arifuzzaman Sardar, Ramen Kumar Biswas, Prolath Dev Nath, " Performance Analysis of Client Side Encryption Tools " , International Journal of Advanced Computer Research (IJACR), Volume-4, Issue-16, September-2014 ,pp.888-897. 